\title{
The modification of tidal ellipses by stratification in the Rhine ROFI
}

\author{
A. J. SOUZA* and J. H. SIMPSON*
}

(Received 6 June 1994; revised 17 November 1994; accepted 24 April 1995)

\begin{abstract}
We report on recent observations of the vertical structure of density and velocity profiles, using a bottom mounted ADCP, in the Rhine ROFI system in the North Sea which confirms previous indications that the presence of stratification modifies the vertical structure of the tidal ellipse characteristics. During periods of stratification, the ellipses change from degenerate to a more circular pattern, with the surface ellipse rotating clockwise and the bottom ellipse rotating anticlockwise. The surface to bottom ellipticity difference $\Delta \varepsilon$ is found to be closely related to a bulk Richardson number which incorporates both the stratification and a measure of the tidal shear. An explanation of the observed dependency of ellipticity on the density structure is offered in terms of the different thickness of the frictional layers for clockwise and anticlockwise motion in a rotating system. The changes in polarization of the flow are large enough to introduce a significant cross-shore velocity component which enhances the vertical shear in the tidal flow and is responsible for the strong semi-diurnal variation of stratification observed in this ROFI system.
\end{abstract}

\section{INTRODUCTION}

In a previous paper (Simpson et al., 1993) we have described the Rhine ROFI (Region Of Freshwater Influence) system, near the Dutch coast, which is maintained by an average discharge of $2200 \mathrm{~m}^{3} \mathrm{~s}^{-1}$ by the Rhine into the North Sea. This input is a major source of buoyancy which modifies the structure and dynamics over a large area of the south-eastern North Sea. Under the influence of the earth's rotation, this buoyancy input tends to drive a coastally-trapped current flowing to the north-east which is generally in the same direction as the residual flow due to wind forcing and tidal rectification.

The processes of stratification and mixing in such a ROFI system have been discussed by Simpson et al. (1990a). The basic stratifying influence of the estuarine circulation is in competition with the stirring by tidal flows, winds and waves. This competition between stabilizing and stirring forces in a non-rotating system is clearly demonstrated in the laboratory experiments of Linden and Simpson (1988), but in the case of the Rhine ROFI, the effect of rotation is also apparent in limiting the spreading of the density current and deflecting it into the north-flowing coastal current. At the same time, the interaction of tidal shear with the density field induces semi-diurnal oscillations of stratification in the ROFI. Variations in tidal shear and hence in the forcing of this periodic stratification have recently been linked to changes in the ellipse configuration (Simpson and Souza, 1995).

Studies of the velocity profile in shallow waters, have shown that the ellipse properties of

${ }^{*}$ School of Ocean Studies, University College of North Wales, Menai Bridge, Gwynedd LL59 5EY, U.K. 
tidal currents generally change with depth. This has been successfully explained for the homogeneous case in terms of the bottom Ekman layer dynamics (e.g. Prandle, 1982). The greater length scale of the boundary layer associated with clockwise motion (in the Northern hemisphere) causes the currents to become increasingly clockwise with increasing height above the seabed. Recent observations (Maas and van Haren, 1987; Lwiza $e t$ al., 1991; Visser et al., 1994) suggest that the presence of stratification modifies the tidal dynamics and produces significant deviations from the predicted profiles for the homogeneous rotating case (Prandle, 1982). In this paper, we report on new observations in the Rhine ROFI with a bottom mounted ADCP which demonstrate the direct influence of stratification on the tidal ellipse shape. We show how these changes may also be readily understood in terms of properties of boundary layer theory and deduce a relationship of the ellipticity to the bulk Richardson number.

\section{OBSERVATIONS AND ANALYSIS}

From 2 to 17 September 1992, observations of water column stability and flow were carried out in the Rhine ROFI using an array of five moorings. The array formed a square of $13 \mathrm{~km}$ by $13 \mathrm{~km}$ and had a central mooring at about $16 \mathrm{~km}$ off-shore from Noordwijk (Fig. 1). Each mooring was equipped with four current meters at depths of approximately $1,10,13$ and $16 \mathrm{~m}$; each of which were capable of measuring temperature and conductivity. Acoustic Doppler Current Profilers (acoustic frequency $1 \mathrm{MHz}$ ) were also deployed at moorings $\mathrm{A}$ and $\mathrm{B}$ and we have used the current profile data from these two positions, in conjunction with the temperature and salinity time series to study the controls on ellipse properties. The ADCPs were located in $20 \mathrm{~m}$ of water and cover $80 \%$ of the water column from $3 \mathrm{~m}$ above the bottom to $3 \mathrm{~m}$ below the surface at vertical intervals of $1.5 \mathrm{~m}$.

The harmonic method was used to separate the main tidal constituents of the current velocities. This consists in fitting a tidal function:

$$
T(t)=Z_{0}+\sum_{N} H_{\mathrm{n}} f_{\mathrm{n}} \cos \left\{\sigma_{\mathrm{n}} t-g_{\mathrm{n}}+\left(V_{\mathrm{n}}+u_{\mathrm{n}}\right)\right\}
$$

to each velocity component by a least squares procedure to determine the amplitude, the phase $H_{\mathrm{n}}, g_{\mathrm{n}}$ and the mean $Z_{0}$. The parameters $f_{\mathrm{n}}$ and $u_{\mathrm{n}}$ are the nodal adjustments to the amplitude and phase respectively while $\sigma_{\mathrm{n}}$ and $V_{\mathrm{n}}$ are the tidal frequency and equilibrium phase angle for the constituent, respectively.

In order to observe the daily changes in the vertical structure of the tidal currents, in response to the variations in water column stability, a moving average $M_{2}$ least square fit was carried out for each $25 \mathrm{~h}$ period of the data.

From the amplitude and phase of the $u$ and $v$ components of $M_{2}$ we construct the clockwise and anticlockwise rotary components according to:

$$
\begin{gathered}
U_{\mathrm{ac}}=\frac{1}{2}\left\{u^{2}+v^{2}+2 u v \sin \left(g_{\mathrm{v}}-g_{\mathrm{u}}\right)\right\}^{1 / 2} \\
U_{\mathrm{c}}=\frac{1}{2}\left\{u^{2}+v^{2}-2 u v \sin \left(g_{\mathrm{v}}-g_{\mathrm{u}}\right)\right\}^{1 / 2} \\
g_{\mathrm{ac}}=\tan ^{-1}\left\{\frac{v \cos g_{\mathrm{v}}-u \sin g_{\mathrm{u}}}{u \cos g_{\mathrm{u}}+v \sin g_{\mathrm{v}}}\right\} \\
g_{\mathrm{c}}=\tan ^{-1}\left\{\frac{v \cos g_{\mathrm{v}}+u \sin g_{\mathrm{u}}}{u \cos g_{\mathrm{u}}-v \sin g_{\mathrm{v}}}\right\}
\end{gathered}
$$




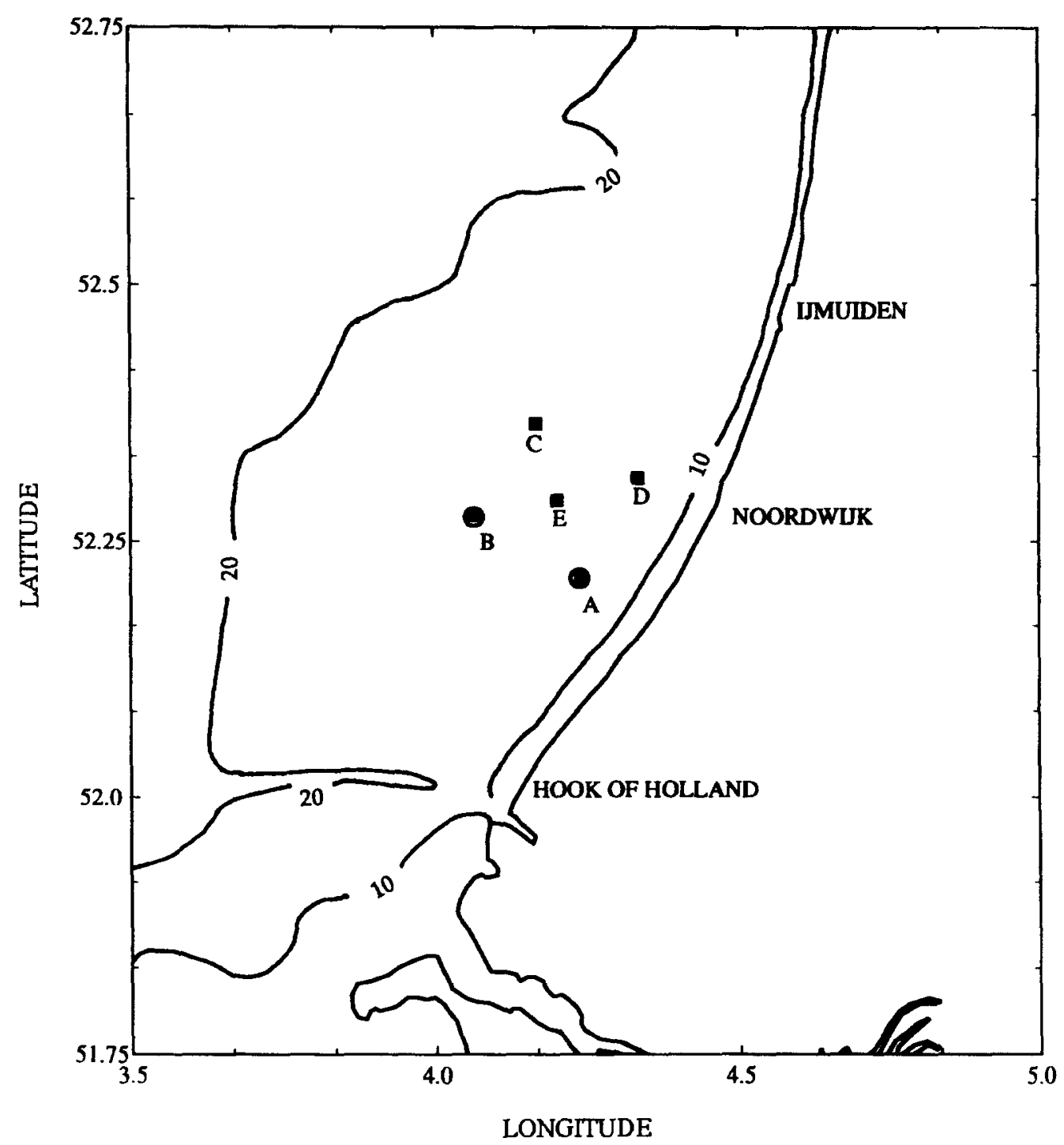

Fig. 1. Rhine ROFI study area with bathymetry in metres and mooring positions ( $\mathbf{D})$. The circles represent the positions where $1 \mathrm{MHz}$ bottom-mounted ADCPs were available.

These rotary components are then used to determine the tidal ellipse properties (Godin 1972):

$$
\begin{aligned}
M & =U_{\mathrm{ac}}+U_{\mathrm{c}} \\
m & =U_{\mathrm{ac}}-U_{\mathrm{c}} \\
\psi & =\left(g_{\mathrm{ac}}+g_{\mathrm{c}}\right) / 2 \\
\phi & =-\left(g_{\mathrm{ac}}-g_{\mathrm{c}}\right) / 2 \\
\varepsilon & =m / M
\end{aligned}
$$


where $U$ and $g$ are the amplitude and phase of the rotary vectors, the suffices ac and c, mean anticlockwise and clockwise respectively; $M$ is the semi-major axis while $m$ is the semi-minor axis of the ellipse, $\psi$ and $\phi$ are the orientation (positive anticlockwise from the east) and phase angles of the semi-major axis.

The ellipticity $\varepsilon$ is defined as the ratio of the semi-minor axis to the semi-major axis; the ellipticity is equal to zero when the ellipse becomes a degenerate and $\varepsilon=1$ when the ellipse is a circle, while the sign give the sense of rotation (i.e. positive anticlockwise and negative clockwise).

\section{RESULTS}

The evolution of stratification $\Delta \rho$ at mooring $\mathrm{A}$ is presented in Fig. 2 together with estimates of tidal and wind stirring, calculated using observations with efficiencies from Simpson and Bowers (1981). Strong bursts of wind stirring with energy inputs of up to 0.2 $\mathrm{mW} \mathrm{m}^{-3}$ are seen to dominate at times over the tidal contribution which increases from a low level around neap tides, near the start of the record, to a peak value of $0.1 \mathrm{~mW} \mathrm{~m}^{-3}$ on day 257. The influence of the combined stirring power inputs is reflected in the mean level of the stratification [Fig. 2(c)]: low stratification at the start of density record follows a bout of strong wind mixing after lower winds and neap tides allow the development of strong stratification at the mooring with values of the mean bottom to surface density difference $\Delta \rho \sim 2 \mathrm{~kg} \mathrm{~m}^{-3}$ on days 254-255. Immediately following this the combination of an episode of strong wind stirring with substantial tidal stirring induces an almost complete vertical mixing of the water column.

Superimposed on the changes in the average level of stratification is a very prominent semi-diurnal variation [Fig. 2(d)] whose amplitude is large in relation to the daily mean so that, at the time of minimum stratification, the system approaches complete homogeneity.

An example of mean stratification over two tidal cycles is presented in Fig. 3, the mean was calculated using 14 repeated sections of a line in front Noordwijk from 5 to $30 \mathrm{~km}$ offshore. The survey was carried out between day 260 and 261 using the undulator SEAROVER which was able to generate one vertical profile every $300 \mathrm{~m}$. The section suggest a stratification of about $\Delta \rho \sim 0.5 \mathrm{~kg} \mathrm{~m}^{-3}$ and the depth of the pycnocline at about 7 $\mathrm{m}$. Although this average section does not present a 2 layer structure due to strong smoothing during the averaging process, the system appears to be two-layer at least during part of the tidal section.

The stratification time series [Fig. 4(a)] indicates three periods in which significant stratification was present at mooring A: (i) from day 250 to 252, (ii) between days 254 and 255 and (iii) from day 256 to day 259 . In between these periods, almost complete mixing prevailed and there was very little vertical structure in ellipticity and ellipse orientation (Fig. 4). The surface ellipses were almost degenerate $(\varepsilon \sim 0)$ with increasing anticlockwise ellipticity at the bottom to values of the order of 0.1 .

By contrast, during the periods of stratification, the surface ellipses acquired marked clockwise rotation with $\varepsilon \sim-0.2$ while in the lower layer the motion became more anticlockwise with $\varepsilon>+0.2$. The transition between the two regimes is well defined and occurs at a depth of $\sim 8 \mathrm{~m}$ which appears to be related to the mean depth of the pycnocline as shown in Fig. 3.

Stratification was also observed to exert some influence on the ellipse orientation as indicated in Fig. 4(d). When the water column is mixed the surface and bottom ellipses are 

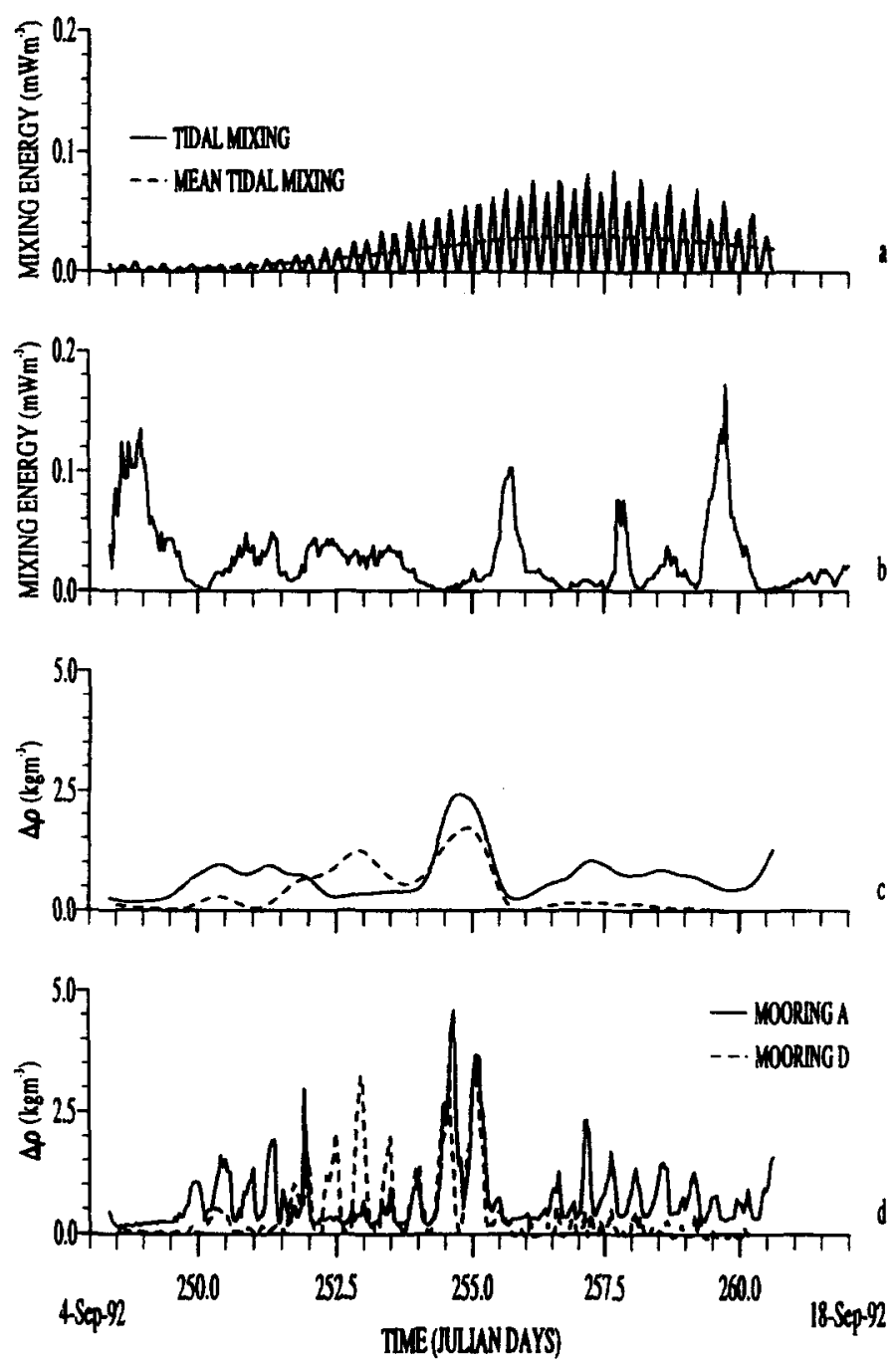

Fig. 2. Mooring A time series. (a) Tidal stirring instantaneous value (dashed) and (b) daily mean (continuous) computed from the velocity of the first bin of the ADCP ( $4 \mathrm{~m}$ above the bed). Wind stirring calculated using data from the Noordwijk tower. (c,d) Stratification $\Delta \rho$, density difference between $16 \mathrm{~m}$ and $1 \mathrm{~m}$ depth as daily mean (c) and instantaneous (d). Mean water depth $20 \mathrm{~m}$.

oriented in the same direction to within $5^{\circ}$, but, when the water column stability develops, there is a bottom surface orientation difference of up to $15^{\circ}$ with the bottom maximum currents being deflected to the west relative to the surface currents.

In the case of mooring B (Fig. 5), where stratification was negligible throughout the observational period [Fig. 5(a)], the vertical profile of tidal currents generally conform qualitatively to the theory for the homogeneous case (i.e. Prandle, 1982). There is little vertical structure at any time; the ellipses are close to degenerate through the entire water column, with slightly stronger anticlockwise rotation near the bottom $(\Delta \varepsilon \sim 0.05)$ [Fig. 


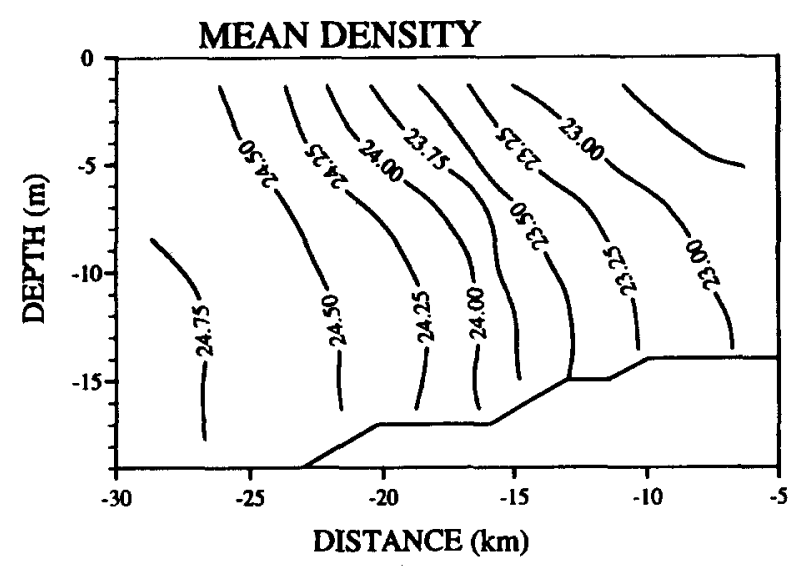

Fig. 3. Mean cross-shore profile of density structure at a cross-shore line in front of Noordwijk. The average was calculated using 14 repeated sections, using the SEAROVER undulator, over 2 $M_{2}$ tidal cycles.

5(b)]. These results for the homogeneous case act as a valuable control in identifying the particular effects of stratification on the form of the tidal ellipses.

\section{MODIFICATION OF THE TIDAL ELLIPSE BY STRATIFICATION}

To understand the contrasting behaviour observed under mixed and stratified conditions we need to examine the problem of tidal flow in two directions on a rotating frame (the Earth) under the influence of frictional stresses. The motion, in which the particle trajectories are generally elliptical in form, may usefully be decomposed into anticlockwise and clockwise components. The characteristic boundary layer thickness for these two components is then given by (Prandle, 1982):

$$
\delta_{-} \simeq\left(\frac{2 N_{\mathrm{z}}}{(\omega-f)}\right)^{1 / 2}
$$

for clockwise motion and

$$
\delta_{+} \simeq\left(\frac{2 N_{z}}{(\omega+f)}\right)^{1 / 2}
$$

for anticlockwise motion, where $\omega$ is the frequency of the tidal constituent concerned, $N_{\mathrm{z}}$ is the eddy viscosity and $f$ the Coriolis parameter. Outside these bottom boundary layers the tidal oscillation is not significantly influenced by frictional stresses and is depth independent.

At the latitude of the Rhine ROFI, $f \sim 1.15 \times 10^{-4} \mathrm{~s}^{-1}$ so that with $\omega=1.41 \times 10^{-4} \mathrm{~s}^{-1}$, we have:

$$
\frac{\delta_{+}}{\delta_{-}}=\left(\frac{\omega-f}{\omega+f}\right)^{1 / 2} \simeq 0.3
$$

For the specific case of the Rhine ROFI, the eddy viscosity is not well determined but 

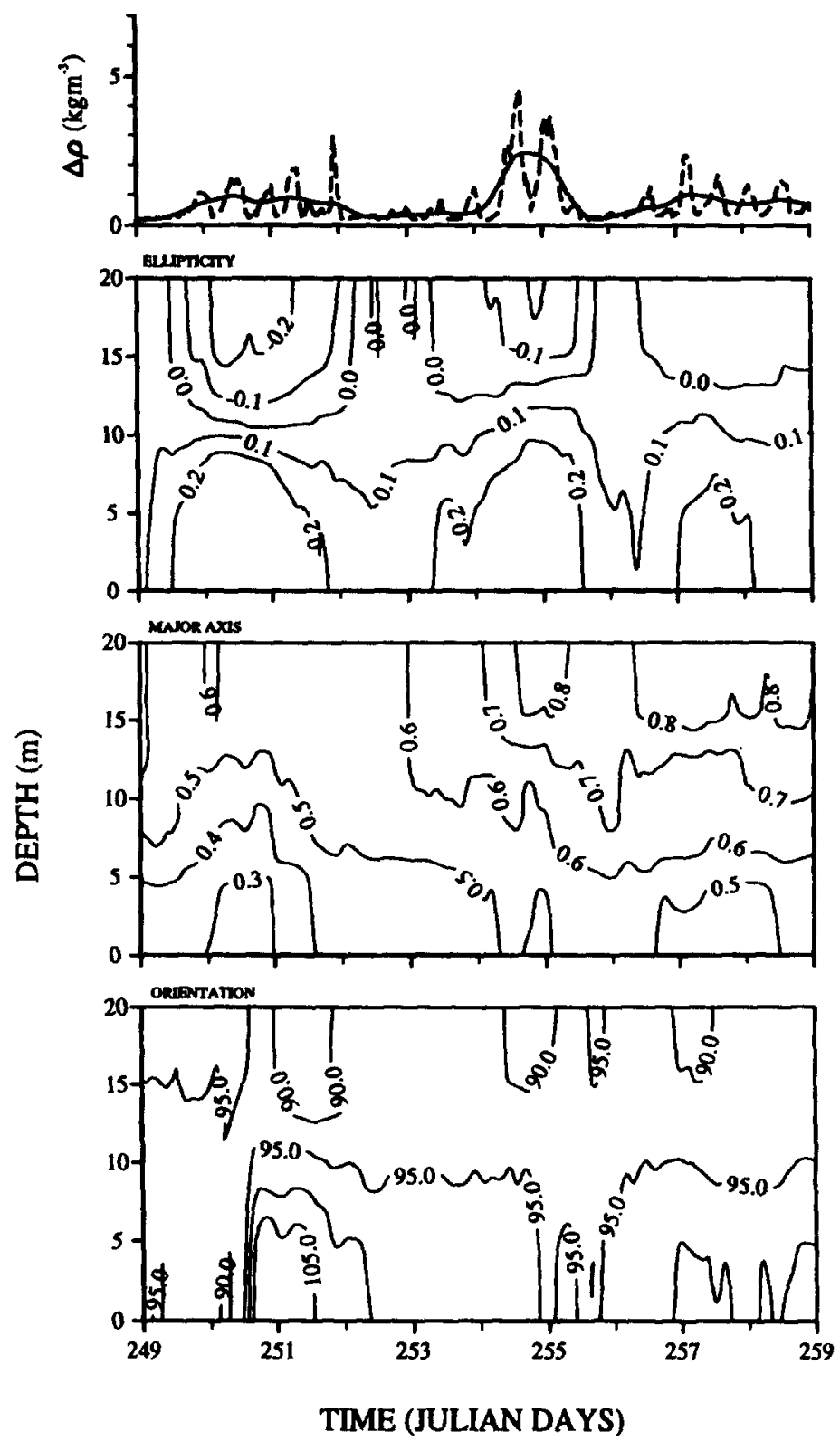

Fig. 4. Time series of ellipse characteristics and water column stratification for mooring A (water depth $20 \mathrm{~m}$ ). (a) The density difference between bottom and top current meter; the continuous line represents the mean stratification averaged over two tidal periods while the dashed line is the instantaneous value. (b) Ellipticity $\varepsilon$ from bottom mounted ADCP data. (c) Semi-major axis of the ellipse in $\mathrm{m} \mathrm{s}^{-1}$ (d) Orientation of the ellipse major axis in degrees (positive anticlockwise from the east). 


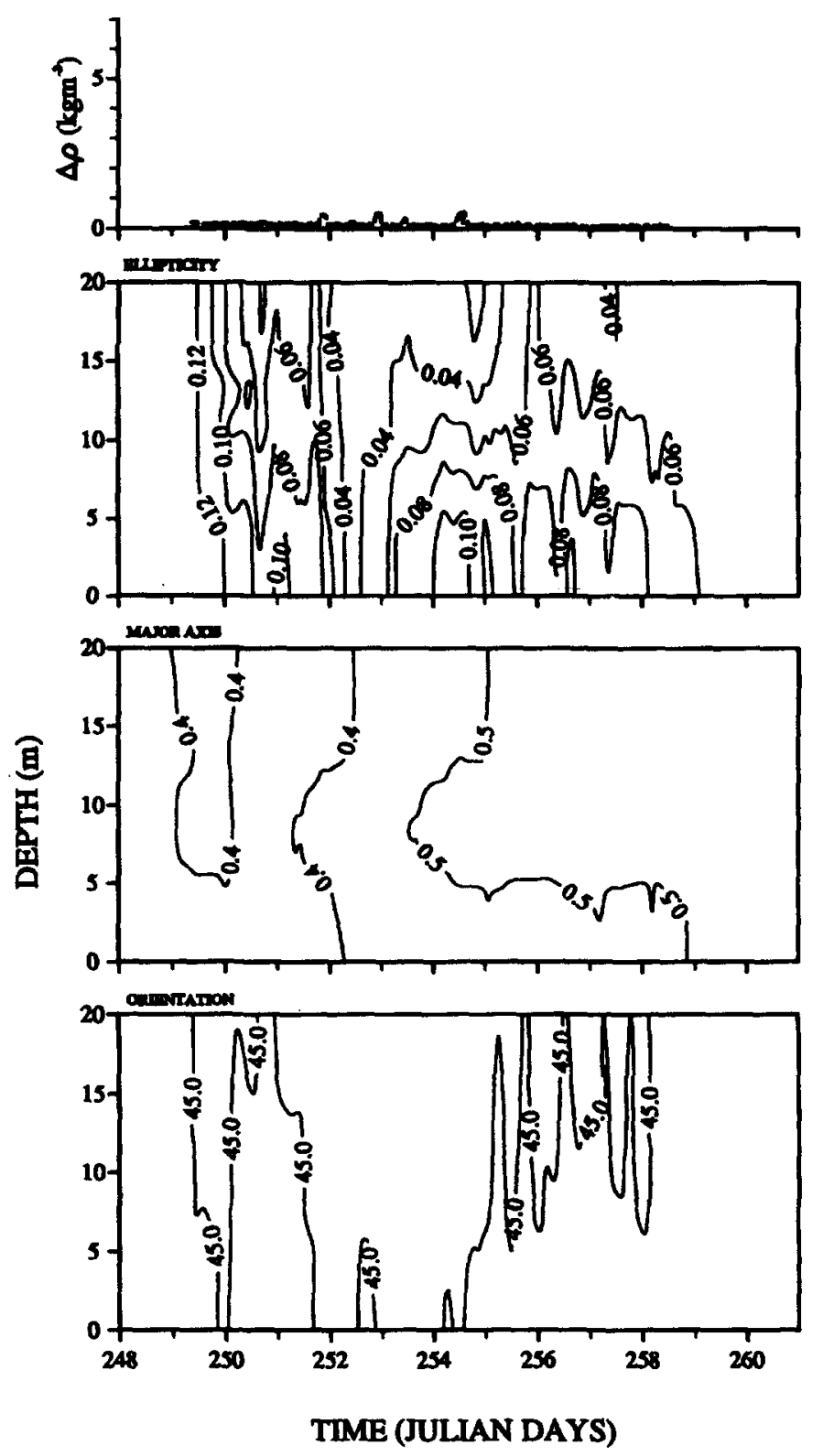

Fig. 5. Mooring B time series (water depth $20 \mathrm{~m}$ ).

approximations using $N_{\mathrm{z}}=\frac{1}{2} k_{\mathrm{b}} \bar{U} h$ (Prandle, 1982), suggest that $N_{\mathrm{z}}=1.2 \times 10^{-2} \mathrm{~m}^{2} \mathrm{~s}^{-1}$ which leads to $\delta_{-}=30 \mathrm{~m}$ and $\delta_{+}=10 \mathrm{~m}$. Hence the thickness of the frictional layer for the clockwise component will cover the entire water column, while the anticlockwise component extends to only half of it.

The very different scales for the clockwise and anticlockwise boundary layers accounts for the general tendency for rotation of the current ellipse to become more clockwise with 
(a) HOMOGENEOUS
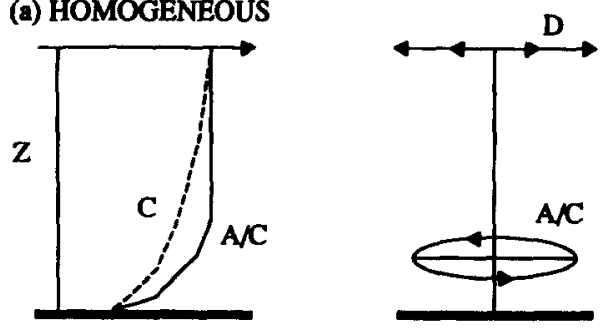

(b) STRATIFIED
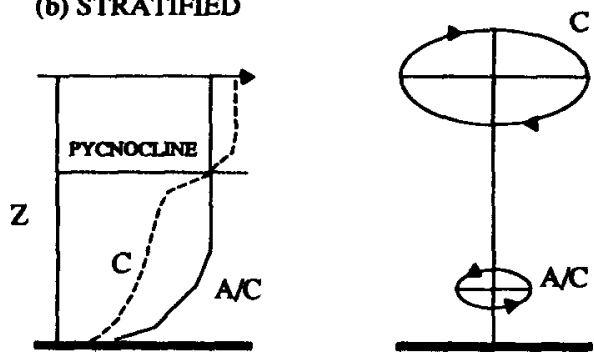

Fig. 6. Schematic of the vertical profile of the clockwise and anti-clockwise rotary components and ellipse configuration. (a) For the homogeneous case when $N_{\mathrm{z}}$ is constant. (b) For the stratified case when $N_{\mathrm{z}}$ is reduced at the pycnocline.

increasing distance from the seabed. Figure 6(a) illustrates this effect for the case of equal clockwise and anticlockwise components at the surface which produce a degenerate ellipse with rectilinear tidal flow. Near the bed the boundary layer structure reduces the clockwise component $U_{\mathrm{c}}$ relative to the anticlockwise $U_{\mathrm{ac}}$ so that the resultant ellipse exhibits anticlockwise rotation.

Consider now what happens when the water column becomes stratified while the tidal forcing remains unchanged [Fig. 6(b)]. The effect of water column stability is to reduce the eddy viscosity $N_{\mathrm{z}}$ in the vicinity of the pycnocline; this suppresses turbulent motions within the pycnocline and inhibits the transfer of momentum. The clockwise component acquires its "free-stream" value above the pycnocline while the frictional forces intensify below the pycnocline. The effect on the anticlockwise component is negligible due to the fact that it is already close to its free-stream value at the depth of the pycnocline. In terms of the tidal ellipses, the surface current ellipses thus acquires a clockwise rotation, while the near bottom current ellipse becomes more anticlockwise due to a reduction in the clockwise component associated with the concentration of frictional influence below the pycnocline.

\section{SUMMARY AND DISCUSSION}

The observations of the polarization of tidal currents reported here are consistent with the above explanation in terms of boundary layer theory. The essential mechanism, which has also been demonstrated in two layer models for example by Maas and van Haren (1987) and Visser et al. (1994), is that changes in $N_{z}$ due to stability and stirring control the level of coupling between surface and bottom layers and that this change in coupling influences the clockwise and anticlockwise motions to a different extent. If this basic 


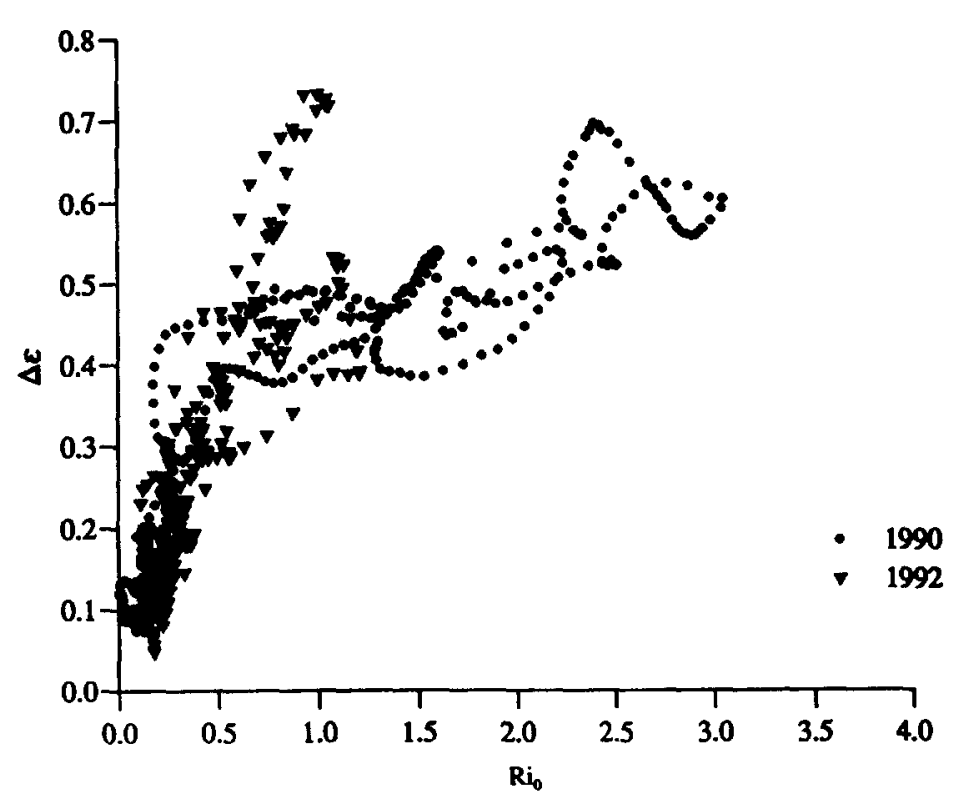

Fig. 7. Relationship between bottom-surface ellipticity difference $(\Delta \varepsilon)$ and bulk Richardson number $R i_{\mathrm{o}}$ inside the stratified area. Mooring $\mathrm{A}$ on September 1992 data ( $\left.\boldsymbol{\nabla}\right)$ and Mooring B on October (Jdays 280 to 290) 1990 data (*) (see Simpson et al. (1993) for location).

explanation of our observation in terms of boundary layer dynamics is correct, we might expect that there should be a consistent relationship between the changes in polarization through the water column and the levels of water column stability and stirring. Such a possibility is also suggested by the close correlation of the changes in $\varepsilon$ and stratification apparent in Fig. 4.

We have tested for such a general relationship by plotting the surface to bottom contrast in ellipticity versus a bulk Richardson number defined as:

$$
R i_{\mathrm{o}}=\frac{g h \Delta \rho}{\rho U^{2}}
$$

where $\Delta \rho$ is the surface to bottom density difference and $U$ is the amplitude of the tidal current, which varies over the spring-neaps cycle. The results (Fig. 7) show that there is a reasonably consistent relationship between $\Delta \varepsilon$ and $R i_{\mathrm{o}}$ although with greater variability at larger Richardson numbers.

To further explore this idea, we have also plotted, in the same form, the equivalent data from earlier current meter observations made in September and October 1990 (Visser et $a l ., 1994)$. The results cover a wider range of $R i_{\mathrm{o}}$ but indicate the same form of relationship with closely comparable slopes for $R i_{\mathrm{o}}<0.5$. At higher $R i_{\mathrm{o}}$, there are indications that the slope decreases with $\varepsilon$ eventually reaching an upper limit. As $R i_{\mathrm{o}}$ increases from zero stratification starts to decouple the bottom and surface layers until, $R i_{\mathrm{o}}=0.5$, the decoupling is almost completed. Further increase in stratification will have less effect on the ellipticity difference because the clockwise velocity has almost reached its free-stream value in the surface layer. Increasing scatter at higher $R i_{\mathrm{o}}$ may be due to differences in the form of stratification (e.g. the depth and strength of the pycnocline) which are not reflected in the bulk density difference component of $R i_{\mathrm{o}}$. Similarly some variability will be 
attributable to variations in wind stirring which is not included in the shear component of $R i_{\mathrm{o}}$. This deviation is apparent in the 1992 data where there are high values of ellipticity for $R i_{\mathrm{o}}$ values between 0.5 and 1.0. These anomalously high $\varepsilon$ values correspond to the first period of stratification on days 250-252. During this period tidal mixing is negligible and wind stirring is moderate, resulting in a deeper pycnocline.

It is possible that temporal variability in the ellipse characteristics may arise in our method of analysis through the interaction of the $M_{2}$ and $S_{2}$ constituents. Such changes would, however, affect the strongly mixed area (Fig. 5) in the same way as areas liable to stratification (Fig. 4). The striking differences between Figs 4 and 5, in ellipticity and orientation, indicate that the observed changes in $\Delta \varepsilon$ in Fig. 4 are due to the effect of stratification.

The difference in the amplitude of the major axis is related to the fact that the $M_{2}$ tide in the Dutch coast behaves as a progressive Kelvin wave in which the velocity amplitude will decrease as we move further away from the coast. The changes which could be attributed to the interaction of $M_{2}$ and $S_{2}$ tidal constituents are temporal variations in the amplitude and of the phase through the spring-neaps cycle (see for example Simpson et al., 1990b). The amplitude of our pseudo- $M_{2}$ semi-major axis is equal to $\left(M_{2}+S_{2}\right)$ at spring tides and $\left(M_{2}-S_{2}\right)$ at neap tides as observed in Figs 4 and 5. The effect of the changes of ellipticity observed in Fig. 4 do not appear to be related to the spring-neap cycle, because in this case windstress dominates over tidal stirring in the control of stratification.

Finally we should remark that, as well as being of interest in relation to our understanding of tidal dynamics, these observed changes in polarization are also responsible for inducing a strong component of vertical shear in the cross-shore tidal flow at times of stable stratification. The consequent straining of the density field induces a strong semi-diurnal variation in the stratification which is a characteristic of the region and can result in a brief episode of almost complete vertical mixing on each tidal cycle [Fig. 2(d)].

Acknowledgements-This study was supported by the EEC through the Mast program under the MAS2-CT930054 contract. A. J. Souza wishes to thank CONACyT, Mexico for the studentship provided.

\section{REFERENCES}

Godin G. (1972) The Analysis of Tides. University of Toronto Press, $264 \mathrm{pp}$.

Linden P. F. and J. E. Simpson (1988) Modulated mixing and frontogenesis in shallow seas and estuaries. Continental Shelf Research, 8, 1107-1127.

Maas L. R. M and J. J. van Haren (1987) Observation on the vertical structure of tidal and inertial currents in the central North Sea. Journal of Marine Research, 45, 293-318.

Prandle D. (1982) The vertical structure of tidal currents. Geophysics and Astrophysics Fluid Dynamics, 22, 2949.

Simpson J. H., J. Brown, J. Matthews and G. Allen (1990a) Tidal straining, density currents and stirring control of estuarine stratification. Estuaries, 13(2), 125-132.

Simpson, J. H., E. G. Mitchelson-Jacob and A. E. Hill (1990b) Flow structure in a channel from an acoustic Doppler current profiler. Continental Shelf Research, 10(6), 589-603.

Simpson J. H., W. G. Bos, F. Schirmer, A. J. Souza, T. P. Rippeth, S. E. Jones and D. Hydes (1993) Periodic stratification in the Rhine ROFl in the North Sea. Oceanologica Acta, 16, 23-32.

Simpson J. H. and A. J. Souza (1995) Semi-diurnal switching of stratification in the Rhine ROFI. Journal of Geophysical Research, 100(4), 7037-7044.

Simpson J. H. and D. G. Bowers (1981) Models of stratification and frontal movements in the shelf areas. DeepSea Research, 28A, 727-738.

Visser A. W., A. J. Souza, K. Hessner and J. H. Simpson (1994). The influence of water column stratification on tidal profiles in a ROFI system. Oceanologica Acta, 17(4), 369-381. 